\title{
Una lectura del transporte público concesionado en Ciudad de México desde la visión del orden urbano ${ }^{1}$
}

\section{A Reading Public Transport Concession in Mexico City from the Perspective of Urban Order}

\author{
Uma leitura do transporte público \\ concessionado na Cidade do México desde a \\ visão da ordem urbana
}

\section{Madisson Yojan Carmona Rojas ${ }^{2}$}

Docente investigador de la Universidad Pedagógica Nacional de Colombia, Bogotá, Colombia Docente de la Universidad de Cundinamarca, Bogotá, Colombia mycarmonar@pedagogica.edu.co

Recibido: $23 / 08 / 2015$ Aprobado: 06/05/2016

1 Este artículo hace parte del desarrollo de la tesis de Maestría en Ciencias Sociales y Humanidades titulada: Orden urbano y Metrobús en la ciudad de México. El caso de la línea 1, presentada en diciembre de 2014. Para ello, se contó con el apoyo del programa de becas de posgrado de la Universidad Autónoma Metropolitana y del Consejo Nacional de Ciencia y Tecnología-Conacyt- de México.

2 Maestro en Ciencias Sociales y Humanidades. 


\section{Resumen}

El presente artículo propone una lectura del transporte público concesionado de Ciudad de México desde la perspectiva de análisis del orden urbano -concepto desarrollado por Emilio Duhau y Ángela Giglia en diversos estudios-. Para ello, se definen cinco operadores espaciales a partir de los cuales entrará en diálogo la estructura funcional del transporte concesionado con el ámbito normativo y las prácticas espaciales de los usuarios de las rutas de autobuses. De esta forma, es posible tener una visión del transporte público de Ciudad de México que, alejándose de la mirada tradicional del caos, reconozca la existencia tanto de continuidades como de rupturas en la estructura de este servicio público. Este enfoque de trabajo propuesto busca aportar a la comprensión del proceso de implementación del sistema de transporte Metrobús (Bus Rapid Transit) que opera en la ciudad desde hace diez años.

Palabras clave: transporte público concesionado; Ciudad de México; orden urbano; operador espacial; Bus Rapid Transit.

\section{Abstract}

This article proposes a reading of public transport under concession in Mexico City from the perspective of urban order analysis -a concept developed by Emilio Duhau and Angela Giglia in various studies. To do this, five spatial operators are defined from which the functional structure of the transport under concession will dialogue with regulations and spatial practices of the users of bus routes. Thus, it is possible to have a vision of public transport in Mexico City that recognize the existence of both continuities and ruptures in the structure of this public service, away from the traditional look of chaos. This working approach seeks to contribute to understanding the implementation process of Metrobús transport system (Bus Rapid Transit) operating in the city for ten years ago.

Keywords: public transport concession; Mexico City; urban order; spatial operator; Bus Rapid Transit.

\section{Resumo}

Este artigo propõe uma leitura de concessão de transporte público da Cidade do México a partir da perspectiva de análise ordem urbana de um conceito desenvolvido por Emilio Duhau e Angela em vários estudos de Giglia. Para fazer isso, os operadores definidos cinco espaciais a partir do qual entrar em diálogo a estrutura funcional do ambiente regulatório concessão de transporte e práticas espaciais dos usuários das linhas de ônibus. Assim, é possível ter uma visão do transporte público na Cidade do México, longe do olhar tradicional do caos, reconhecer a existência de ambas as continuidades e rupturas na estrutura deste serviço público. Esta abordagem de trabalho proposto visa contribuir para a compreensão do processo do sistema de transporte Metrobús (Bus Rapid Transit) implementação na cidade há dez anos.

Palavras-chave: concessão de transportes públicos; Cidade do México; ordem urbana; o operador espaço; Bus Rapid Transit. 


\section{Introducción}

La llegada del BRT (Bus Rapid Transit) a Ciudad de México representó importantes transformaciones en cuanto al funcionamiento y organización de las rutas que circulaban por los corredores que fueron intervenidos. Sin embargo, este no es el único impacto que se puede apreciar, toda vez que el Metrobús -visto en el plano urbano más amplio-, al ser un sistema con una disposición particular de filtros, controles y regulaciones, ha generado -o por lo menos ha contribuido a ello- nuevas dinámicas de uso y apropiación del espacio que conviene estudiar con detenimiento.

La avenida de los Insurgentes, que es para muchos un ícono de Ciudad de México, fue la primera vía intervenida con el BRT en el 2005. Atraviesa la urbe de norte a sur, y en su recorrido se encuentran zonas residenciales, comerciales y educativas bastante representativas de la capital; lo cual fue uno de los factores decisivos para elegirla como la primera línea del Metrobús. Antes del BRT, circulaban por Insurgentes principalmente unidades de transporte de baja capacidad, concesionadas a la Ruta 2 en un esquema normativo y operativo que necesariamente se transformó para dar paso a los arreglos propios del Metrobús.

Teniendo en cuenta lo anterior, en este artículo se establece, en primer lugar, los elementos conceptuales que permitan leer la configuración del transporte público desde la óptica del orden socio-espacial urbano. En segundo lugar, se precisan los operadores espaciales ${ }^{3}$ sobre los cuales se estructura el sistema BRT de Ciudad de México, para luego pasar a identificar, con base en dichos operadores, el ámbito normativo y las prácticas de los pasajeros asociadas con el modelo de rutas de transporte concesionado en la ciudad.

\section{Orden urbano}

El asunto del orden socio-espacial urbano puede abordarse desde diversas visiones o ángulos de análisis. Existen copiosos estudios que aunque no refieren particularmente este término sí se han encargado de problematizar las relaciones que establecen los habitantes de las urbes con los diferentes espacios que ocupan. La abundante producción sobre el tema proviene de un amplio espectro de tradiciones teóricas y académicas -cada una con su cariz particular-, entre las cuales pueden nombrarse la sociología, la antropología, el urbanismo y la geografía.

Ahora bien, para acercarnos al objetivo del presente trabajo, es pertinente retomar la conceptualización que desarrollan en diversos textos, al respecto del orden urbano, Emilio Duhau y Ángela Giglia, quienes se refieren a este concepto en los siguientes términos: "Un conjunto de normas y reglas tanto formales (pertenecientes a algunas jerarquías del orden jurídico) como convencionales a las que recurren los habitantes de la ciudad explícita o tácitamente en el desarrollo de práctica relacionadas con los usos y formas de apropiación de los espacios y bienes públicos de uso colectivo" (2004, 258). En su libro Las reglas del desorden: habitar la metrópoli, Duhau y Giglia (2008) se encargan de ampliar y complejizar

3 Este término se definirá en el último apartado del presente artículo. 
los elementos descriptivos anteriores, vinculándolos, a su vez, con el concepto de "experiencia metropolitana". En este artículo, es importante hacer referencia a este asunto, en relación con el de orden urbano, toda vez que nos interesa ver cómo con la entrada en funcionamiento del Metrobús, la experiencia de movilizarse por la ciudad -los usuarios particularmente-cambia a partir de un punto de inflexión que no es solo de carácter temporal sino también espacial; un antes y un después del BRT.

El orden urbano, de acuerdo con Duhau y Giglia (2008), no es ni unitario ni inmóvil en el tiempo, por lo tanto, es importante resaltar que es producido por una amplia gama de actores y de fuerzas, que se encuentran en constante tensión. Así, podemos decir que la producción del espacio, y con ello de los usos y formas de apropiación del mismo, es de naturaleza conflictiva. De lo anterior se desprende que cada grupo social o agremiación manifiesta intereses y asuntos espaciales particulares que son movilizados con arreglo a diversos grados de territorialización.

Por lo anterior, en este artículo, se hará referencia tanto a la experiencia urbana como a la dimensión conflictiva de la misma, como factores que contribuyen a alterar el orden urbano, tanto en lo relativo al marco regulatorio como en lo tocante a los usos y prácticas de apropiación del espacio en las ciudades.

\subsection{El marco regulatorio}

Como se desprende de la definición de orden urbano, las diversas prácticas sociales sobre el espacio público en las ciudades tienen correspondencia con lo que podríamos denominar marco regulatorio urbano, el cual opera como factor que posibilita o restringe el margen de acción de los habitantes. Así, el establecimiento de normas de tipo formal o convencional (Duhau y Giglia 2004, 262) puede entenderse como una necesidad histórica de ordenar tanto el espacio como las formas de uso y apropiación del mismo.

Dichas normas, cualquiera que sea su naturaleza, han obedecido, a lo largo del tiempo, a diversas lógicas relacionales que involucran tanto a las autoridades de gobierno como a los habitantes y demás actores interesados en el desarrollo urbano. De allí que sea importante precisar que el acomodo y la proporción de influencia de estos mismos sectores de la sociedad en la configuración normativa ha sido variable $y$, por lo mismo, sus intereses han sido movilizados atendiendo a condiciones políticas, económicas y culturales presentes en cada periodo histórico.

Ahora bien, no basta con resaltar que tanto el carácter formal como el convencional de las normas que regulan el orden urbano obedece a relaciones históricas -de contraposición o de colaboración- de intereses manifestados por actores, sino que es importante, en el mismo tenor, determinar las características propias tanto de lo formal como de lo convencional, tratando de ver, además, sus mecanismos de operación y la manera como han contribuido -por acción u omisión- en la configuración del panorama de la ciudad informal.

Tomando en consideración lo dicho, el aspecto formal del marco regulatorio corresponde -en la normativa jurídica- al conjunto de decretos, leyes, programas y planes de carácter reglamentario que buscan ordenar "la apropiación del suelo 
y sus usos" (Duhau y Giglia 2004, 264). Esta serie de normas formales son promulgadas por instancias de gobierno encargadas de definir el ámbito de aplicación de las mismas, así como las condiciones de tiempo y espacio para su presentación, ejecución y cumplimiento.

Por consiguiente, en el campo de acción de la normatividad urbana es posible encontrar diversos niveles de actuación que corresponden, igualmente, a ámbitos o escalas diferenciadas. Ello indica que el ordenamiento legal describe una trayectoria gradual de elaboración que va desde lo más amplio y complejo, hasta lo más concreto. Así, en el Distrito Federal encontramos como marco reglamentario general la Ley general de desarrollo urbano de 1996, de la cual se derivan las leyes sectoriales, como la Ley de transporte y vialidad del Distrito Federal de 2002, con sus normas complementarias entre las que se encuentra el Reglamento de tránsito de 1999.

En cuanto al aspecto convencional del marco regulatorio urbano, conviene decir que, contrario a lo que sucede con el anterior, obedece a parámetros que se distancian de lo jurídico, y se instalan en el campo de la interacción cotidiana entre los habitantes de la urbe. En el espacio de la ciudad, por tanto, se generan acuerdos de acción sobre actividades particulares que no se formalizan necesariamente en lo legal, y que están vinculadas "con conocimientos prácticos" que constituyen la experiencia urbana (Duhau y Giglia 2004, 263).

Un ejemplo de lo anterior estaría dado por la existencia en diferentes sectores de la ciudad de personas encargadas de regular el estacionamiento de vehículos en las vías. Comúnmente conocidos como "franeleros" o "viene-viene", son quienes se apropian de un segmento de la vía para permitir que allí se ubiquen los automóviles que no encuentran resguardo o espacio para estacionarse en otros lugares. De lo anterior se deriva una serie de acuerdos informales entre los propietarios de los vehículos y los franeleros, en los que establecen tanto las tarifas por el servicio prestado como las condiciones relativas a la seguridad e integridad del vehículo en caso de hurto, avería o en la eventualidad de la presencia de las autoridades de tránsito del D.F.

Para el caso particular del transporte público se podría mencionar la presencia, a lo largo de la ruta y particularmente en puntos críticos del recorrido, de personas dedicadas a informar a los operarios sobre los tiempos de distancia entre unidades que cubren trayectos sobre estas vías. Estos "checadores de ruta" son un factor que logra tener injerencia directa en la prestación del servicio, dado que con su información el conductor acelera su marcha o la hace más lenta.

Como vemos en los anteriores ejemplos, los mecanismos de producción de estos acuerdos o normas convencionales describen una trayectoria horizontal que parte de la necesidad de generar acuerdos sobre el uso y apropiación de espacios, actividades o artefactos urbanos. Esta lógica de acción de los actores responde en gran medida a vacíos legales que no han logrado ser cubiertos con reglamentaciones formales, aunque en muchos casos, aun existiendo, los habitantes generan, con base en sus "usos y costumbres", entendimientos que, incluso contraviniendo el orden formal, logran ser efectivos y funcionales a sus fines (Giglia 2012, 17). 
Los dos vectores en los que hemos descompuesto el marco normativo urbano son producidos en condiciones institucionales y políticas particulares que sirven como cimiento para estructurar las modalidades de organización espacial que han sobrevenido en las ciudades desde el mismo momento de su fundación. De tal manera, es posible decir que la relación entre lo normativo y lo urbanístico no es unidireccional, sino de doble vía; un proceso constante de influencias y exigencias recíprocas que se vinculan con la existencia de un llamado orden espacial histórico cambiante y abierto a la injerencia de procesos políticos, económicos y sociales de diversa índole (Giglia 2012, 16).

Esta mirada global sobre la problemática del marco normativo nos permite advertir en la emergencia de tendencias y fenómenos urbanos un potente factor que contribuye a movilizar, a su favor, transformaciones sustanciales en la dimensión formal y convencional de las reglas que constituyen uno de los ejes de análisis del orden urbano.

De tal suerte, en primera instancia, los proyectos urbanos requieren para su funcionamiento un reacomodo de la normatividad en el que no basta con promulgar nuevas leyes y decretos, sino que se deben generar marcos institucionales amplios que permitan darles sustento a las propuestas. Vale la pena decir que estos reacomodos normativos no son propios de los nuevos discursos, ni nacen con las tendencias y fenómenos contemporáneos. Basta revisar la literatura existente sobre desarrollo en Ciudad de México para corroborar que con cada proyecto urbano se han dado transformaciones de la normatividad urbana y de los aspectos institucionales existentes hasta el momento.

En síntesis, podríamos decir, teniendo en ello un primer elemento de análisis, que lo que se advierte como producto de las nuevas tendencias y fenómenos urbanos son exigencias normativas precisas que tienden a configurar sistemas con "reglas de uso más rígidas" (Giglia 2012, 19) asentadas sobre mayores controles y filtros, los cuales tienen un impacto directo en el orden urbano visto en su conjunto. Dichas exigencias se manifiestan, por ejemplo, en instrumentos de gestión, en la creación de instancias de control y vigilancia, y en la instalación de dispositivos o artefactos espaciales que contribuyen a prefigurar un orden derivado -y constituyente- del contenido de los proyectos urbanos.

En segunda instancia, es importante abundar en la naturaleza de las escalas de proveniencia de las exigencias normativas que acompañan a los proyectos urbanos contemporáneos, particularmente los que remiten a la movilidad y el transporte. Así, en la actualidad, no es posible explicar el desarrollo de las ciudades acudiendo únicamente a los factores locales de implementación de propuestas de renovación urbana, sino que hace falta contemplar el panorama más amplio o global en el que se inscribe el contenido de las mismas.

Asuntos como la sostenibilidad, la gobernanza urbana, la disminución de emisiones contaminantes, la redensificación de zonas centrales de las ciudades, el derecho a la ciudad, entre otros, surgen como iniciativas de alcance amplio, que circulan en el panorama metropolitano internacional y movilizan intereses, y que se convierten en un ideal normativo urbano de dimensiones globales. No en vano los gobiernos de las ciudades contemplan en sus planes de desarrollo la ejecución de proyectos que, además de satisfacer las necesidades propias de 
cada urbe, cumplan con estándares de calidad externos en cuanto al medio ambiente, al hábitat y las buenas prácticas de gobierno, por citar solo algunos.

Lo anterior pone de relieve la existencia de facto y de jure de redes de ciudades que permiten el intercambio de información por medio de la suscripción de protocolos y la participación en organismos internacionales de financiamiento de proyectos urbanos acordes con las tendencias actuales. En este ambiente, es necesario resaltar la existencia de corporaciones, ONG y organizaciones que brindan asesoría en áreas estratégicas como la movilidad, la cultura urbana, la vivienda y el desarrollo social en su conjunto.

No obstante, la idea que queremos expresar no es la dependencia incondicional de las ciudades en la actualidad a los preceptos globales, sino remarcar la existencia de éstos como uno de los tantos agentes dinamizadores del desarrollo urbano.

De tal suerte, la aproximación hecha a los aspectos normativos del orden urbano, y que ha estado apoyada en la división entre el carácter formal y convencional de los mismos, nos sirve para identificar las características principales del marco regulatorio que gravita alrededor de las prácticas urbanas de ocupación y apropiación del espacio, que luego se verán con claridad en la organización del transporte público antes y después de la llegada del Metrobús. En tal sentido, debe ponerse de relieve el hecho de que los efectos en la espacialidad de la avenida Insurgentes derivados de las transformaciones normativas son apenas un factor parcial del conjunto de las alteraciones en el orden urbano.

En este sentido, para lograr comprender el proceso de transformación del orden urbano, conviene describir y especificar el segundo ámbito en el que, con fines analíticos, este se ha descompuesto. Por lo tanto, en el siguiente acápite nos referimos al asunto de los usos y prácticas de apropiación del espacio público, procurando establecer, además de su definición, los mecanismos de producción de los mismos.

\subsection{Usos y prácticas en el espacio público}

El espacio público ha sido objeto de intensos debates no solo en el seno de la academia, sino también por parte de sectores de gobierno, organizaciones sociales, planeadores y promotores urbanos, que pretenden definir tanto el ámbito material del mismo, como los usos y prácticas que allí deben tener lugar.

Lo anterior lleva a definir el espacio público urbano, en primera medida, como el escenario por excelencia de las interacciones sociales, donde se dan cita las más variadas y diversas prácticas de los distintos grupos sociales que allí se sienten convocados. En palabras de Simmel (2005, 3), es justamente en las metrópolis donde se puede encontrar la multiplicidad de experiencias que configuran el carácter de lo urbano, dada la "concentración de tantas personas con intereses tan diferentes". Esto configura, por lo tanto, "un sistema articulado" de intereses que se manifiestan en lo que se ha denominado como un modo de vida urbano (Wirth 2005).

En segunda medida, el espacio público ha de ser entendido como aquello que "es de utilidad o de interés común a todos" donde, además, "lo público se vuelve progresivamente sinónimo de político" (Rabotnikof 2003, 19) y, por lo tanto, el 
carácter y naturaleza de las interacciones sociales que allí se dan inciden de forma determinante en la configuración de la ciudadanía. La anterior visión es compartida por Jordi Borja $(2003,67)$, para quien el espacio público "supone dominio público y uso social colectivo" como condiciones esenciales para que en las metrópolis contemporáneas sea posible la construcción de ciudadanía.

Estos dos sentidos del espacio público se encuentran vinculados con una dimensión regulatoria y normativa, tanto desde la órbita jurídica o formal, como desde el ámbito convencional. Lo que se busca con ello, por lo tanto, es garantizar la accesibilidad en condiciones de equidad a los bienes y escenarios de uso público en la ciudad, teniendo en cuenta, de otro lado, las características propias de cada uno de ellos.

No obstante la existencia del espacio público como "un concepto jurídico" (Borja 2003, 65) que interviene en el proceso de prefiguración de prácticas y formas de ocupación, este no es el único factor que alienta el uso y transformación del mismo. No en vano el citado Borja dice que "los comportamientos de la gente pueden crear espacios públicos que no lo sean jurídicamente o que no estén previstos como tales" (Borja 2003, 66), con lo cual se pone de relieve la importancia de las prácticas como fuentes productoras de espacialidad en el escenario urbano.

Este espacio que es socialmente producido, y en la misma medida resultado de un "acontecer solidario" entre individuos, se constituye en el crisol en el que se funden y se han transformado las diversas formas de organización territorial de las ciudades a lo largo del tiempo (Santos 2000, 19). Estas formas históricas pueden ser entendidas como la articulación de modelos de desarrollo urbano con formas de gestión y gobierno propias de cada época, junto a la morfología social resultante de las relaciones económicas y culturales de los actores de la urbe.

En tal medida, las prácticas espaciales de los habitantes de la ciudad solo pueden ser leídas en relación con las formas de organización del territorio y con el orden subyacente a ello. Apoyados en el anterior argumento, podríamos ver cómo la experiencia de transitar y movilizarse por Ciudad de México ha estado marcada no solo por la expansión de la misma, sino también por la implementación de diversos modos de transporte que obedecen tanto a necesidades de la urbe, como a tendencias de desarrollo de alcance continental.

El resultado que se advierte es la transformación de los tiempos de desplazamiento de las personas, así como la alteración del grado de accesibilidad a determinados lugares que es favorecida por la expansión de la oferta de transporte; aunque, en la otra cara de la moneda, el déficit de esta oferta impacta de forma negativa en la posibilidad que tienen algunos habitantes, en particular de las zonas periféricas, para acceder a los servicios y equipamientos urbanos.

Para comprender en un sentido más preciso el asunto de las prácticas espaciales, podríamos decir, acudiendo a Roberto Corrêa, que estas se encuentran vinculadas con el principio de la selectividad espacial. Este atributo, según el autor, es resultante de la conciencia y la existencia de la diferenciación espacial, que viene a configurar un principio ordenador del territorio, en el que se hace patente que la distribución de la oferta de equipamientos urbanos no es uniforme, 
y por lo tanto el acceso a ellos, como acotamos anteriormente, es igualmente diferencial (1995; 2007).

Nos dice Roberto Corrêa que "en el proceso de organización de su espacio, el Hombre actúa de modo selectivo. Decide sobre determinado lugar según presente atributos de interés" para sus actividades $(1995,36)$. Esto permite inferir que los habitantes de la urbe, en su acción de movilizarse por ella, se encuentran interactuando en un espacio que se ha configurado alrededor de atributos específicos que se vinculan, a su vez, con contenidos normativos que vendrían a constituir el orden urbano.

De tal suerte, las prácticas espaciales se conjugan con principios espaciales como la proximidad, la conectividad, la accesibilidad, la centralidad y la dispersión, entre otros, conformando una síntesis analítica que se configura, para el caso del transporte y la movilidad urbana, de acuerdo con el esquema 1.

Esquema 1. Modelo general de movilidad urbana

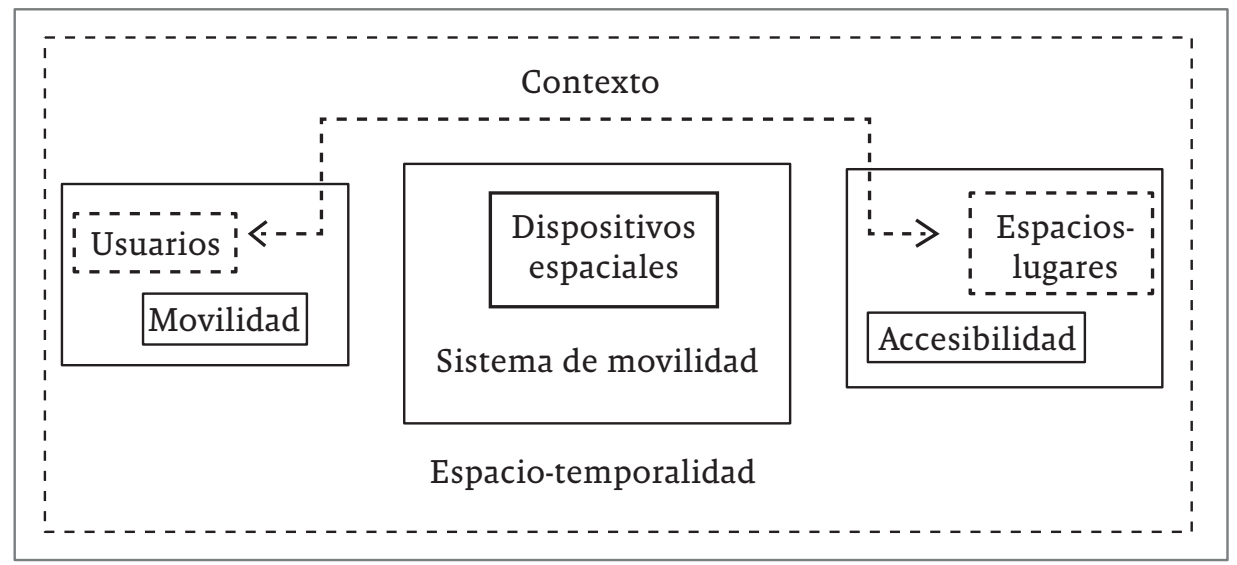

Fuente: Modificado con base en González (2012).

En este esquema encontramos, en primer lugar, a los usuarios quienes son los que se movilizan por la ciudad, empleando para ello, en segundo lugar, diversos dispositivos, artefactos o sistemas de transporte (metro, metrobús, autobuses, vehículos particulares o bicicletas) que permiten o sirven de puente y enlace para, en tercer lugar, acceder a los distintos espacios-lugares de actividad, teniendo en cuenta para este último aspecto tanto las características materiales de estos escenarios, como su usos y destinación; de allí que este conjunto urbano se encuentre determinado por cuestiones relativas a la normatividad o también, como lo acota Borja, "por la dinámica propia de la ciudad y los comportamientos de la gente" $(2003,66)$.

Esta línea de reflexión nos permite ubicar en la relación de los citados principios espaciales con las prácticas urbanas, la matriz de producción del espacio dentro de las ciudades, toda vez que en la permanencia de las actividades cotidianas en el tiempo y en el espacio, se generan procesos de apropiación y se manifiestan representaciones sociales. Así pues, el resultado de las prácticas urbanas, manifestadas en ambas cuestiones -la apropiación y las representaciones-, es la simiente de la experiencia cotidiana de los habitantes de la ciudad. 
Es preciso acotar, por tanto, que la apropiación se encuentra dada, en términos de Perla Serfaty-Garzón (2003, 2), por "una relación de individuos con objetos", en la que el factor mediador entre ambos componentes es el uso que se le da a estos últimos. Es decir, por la vía del uso y acción sobre los objetos, los habitantes de la ciudad intervienen en la construcción de espacio urbano, a la vez que lo incorporan a la experiencia vital que, como hemos visto, es construida por la interacción social.

Cabe resaltar que el sentido de la apropiación descrito por la autora está esencialmente referido a cuestiones de índole "emocional", donde al tomar algo como propio, se está en capacidad de adaptarlo a las necesidades particulares y, por este conducto, "transformarlo en un medio de expresión personal" (Serfaty-Garzón 2003, 2). Reconociendo por lo anterior la naturaleza procesual de la apropiación es posible encontrar, en consecuencia, dos momentos o escenarios particulares que marcan "el sentido y la legitimidad" de la misma (Serfaty-Garzón 2003, 2).

En primera instancia, encontramos las actividades o prácticas espaciales cotidianas de los habitantes de la ciudad. En ellas es pertinente remarcar, por ser nuestro objeto de estudio, la acción de movilizarse diariamente del lugar de habitación a los sitios de trabajo, educación, comercio, recreo y demás, que se encuentra relacionada con el uso y destinación de cada espacio dentro de la urbe, así como con las características de distribución de los lugares de origen y destino de los viajeros. Esta configuración de prácticas, que junto al ámbito normativo constituyen el orden urbano, describe un proceso histórico que da como resultado una estructura específica de ordenamiento del territorio urbano.

En segunda instancia, podemos apreciar que las prácticas espaciales "ancladas en contextos situacionales" del escenario de la ciudad son, junto "con los horizontes de saberes y valores" (Duhau y Giglia 2008, 21), los principales elementos vinculantes que constituyen la experiencia urbana. En ella se manifiestan con claridad "los universos de prácticas y representaciones no solo diferentes sino desiguales en cuanto a las posibilidades de aprovechar el espacio urbano como recurso utilizable, para moverse en él" (Duhau y Giglia 2008, 24).

Cuando nos referimos a la experiencia urbana, se debe dar por sentado que esta no es ni homogénea ni uniforme, dado que se encuentra referida a espacios que tampoco lo son. De allí, pues, que haya sectores de la sociedad capitalina para quienes, por ejemplo, el metro o cualquier medio de transporte público colectivo no es una posibilidad de movilizarse por la ciudad $\mathrm{y}$, menos aun, significa un lugar de encuentro y de sociabilidad.

En tal caso, se puede decir que el ingreso económico es un factor que media en la configuración de la experiencia urbana; sin embargo, este, por sí solo, no logra explicar el porqué de la "selectividad espacial" de Corrêa $(1995,36 ; 2007,69)$ o las "oposiciones espaciales" de Bourdieu $(1999,120)$ que subyacen a la acción de optar por desplazarse acudiendo a un medio de transporte específico. En este orden de ideas, haría falta, por tanto, agregar que los sectores sociales, cualquiera que sean sus posibilidades económicas, desarrollan lo que el mismo Bourdieu $(1999,120)$ ha denominado una "simbolización espontánea" de su quehacer en la ciudad, y que es justamente en ella donde se manifiesta el contenido más 
completo y complejo de la diferenciación socio-espacial en la cual se produce y reproduce el sentido de la experiencia.

La experiencia urbana vendría a estar conformada, entonces, por el resultado de las prácticas espaciales individuales que son socializadas en diversos escenarios de interacción urbana que dependen, asimismo, de condiciones propias de cada contexto espacial, y que remiten a un habitus urbano en el que se condensan dos cuestiones particulares: los contenidos normativos -cualquiera que sea su naturaleza- asumidos por los individuos mediante la repetición -o la coacción-, y la presentación del espacio en formas de "valoración y acción" que en la práctica se manifiestan como recursos particulares de ocupar y habitar la ciudad y, por tanto, de apropiarse de ella (Giménez 2005, 69).

Con esto último llamamos la atención sobre la naturaleza conflictiva del espacio urbano (Duhau y Giglia 2004; Bourdieu 1999) donde, por su misma complejidad, se manifiestan diferencias entre intereses y asuntos, formas de apropiación y uso del mismo, que se activan, por ejemplo, con ocasión de un cambio en algunos de los factores que constituyen el orden urbano.

\section{El orden del transporte público concesionado}

Es indiscutible que el transporte público representa un factor de primordial importancia en la configuración y transformación tanto de la estructura espacial de las ciudades como de la experiencia urbana. Amplios estudios se han dedicado a observar cómo el cambio en los sistemas de transporte -y la tecnología asociada a ellos- ha contribuido a generar o acentuar centralidades, a expandir o controlar la mancha urbana y a lograr que el creciente número de población de la capital tenga la posibilidad de movilizarse por la ciudad con índices aceptables de eficiencia.

Otra parte considerable de estudios se ha orientado a comprender los factores de sociabilidad asociados y derivados de cada uno de los modos de transporte, partiendo de un supuesto esencial: hay diferencias sustanciales en la experiencia de movilidad de los habitantes que son dadas, en gran medida, por el paso del tranvía a los autobuses o por la construcción del metro y por la implementación del BRT. Esto se puede ilustrar con lo que nos refiere Salomón González, al respecto de lo que él llama "el sistema automóvil":

\footnotetext{
Este artefacto (el automóvil) socio-tecnológico tiene implicaciones muy serias en los procesos de territorialización. La reconfiguración del espacio es clara a partir de la masificación del automóvil; las distancias se incrementaron y el paisaje urbano (y suburbano) se transformó para dar acogida a una movilidad basada en el automóvil. Desde el diseño de la vivienda, pasando por la forma y configuración de la traza urbana, hasta la emergencia de nuevos lugares y servicios, son testimonio de la profunda y amplia integración del coche en el modo de vida de millones de personas (González 2012, 2).
}

Puede hablarse, con la implementación de nuevos sistemas de transporte, de cambios en los tiempos de traslado, en las condiciones de seguridad y comodidad de los mismos, o en la posibilidad de acceder con mayor facilidad a determinados lugares de la ciudad, en lo que cabe acotar que la experiencia de 
los usuarios no es la única que se altera, puesto que también existen modificaciones en los arreglos institucionales y normativos que impactan, por ejemplo, en la relación establecida entre los gobiernos y los prestadores del servicio.

Se puede inferir que hay una matriz común a estos grupos de estudios en la cual se hacen visibles los elementos estructurantes del orden urbano. Nos referimos con ello al hecho de que los sistemas de transporte se encuentran configurados, de un lado, por un complejo normativo e institucional y, de otro, por prácticas de movilidad de los usuarios, que tienen lugar en una estructura espacial determinada por factores como el tipo de unidades, la capacidad de las mismas, el uso y la restricción para circular por determinadas vialidades o la existencia de lugares para ascender o descender de los vehículos (Islas 2000; Salazar 2008).

\subsection{La normatividad}

De acuerdo con Víctor Islas, el aspecto normativo y regulatorio de la prestación del servicio de transporte obedece a parámetros jurídicos y a los "aspectos políticos, institucionales y las costumbres que se han forjado en décadas de funcionamiento" $(2000,220)$. Esto nos lleva a pensar en la doble dimensión de lo normativo, en la cual se encuentran los parámetros tanto formales como convencionales que median en las actividades urbanas.

Teniendo en cuenta lo anterior, los marcos reglamentarios formales vigentes en el momento de entrada en funcionamiento del metrobús por Insurgentes, eran los siguientes:

- Ley de Desarrollo Urbano del Distrito Federal, aprobada por la Asamblea de Representantes del Distrito Federal y publicada en la Gaceta Oficial el 29 de enero de 1996.

- Programa General de Desarrollo del Distrito Federal, aprobado por la Asamblea Legislativa del Distrito Federal y publicado en la Gaceta Oficial el 31 de diciembre de 2003.

- Ley de Transporte y Vialidad del Distrito Federal (en adelante LTVDF), aprobada por la Asamblea Legislativa del Distrito Federal y publicada en la Gaceta Oficial el 26 de diciembre de 2002.

- Reglamento de Tránsito del Distrito Federal (en adelante RTDF), aprobado por Asamblea Legislativa del Distrito Federal y publicada en la Gaceta Oficial el 30 de diciembre de 2003.

Cabe acotar que en el Distrito Federal la dependencia oficial encargada de regular el servicio de transporte público de pasajeros, hasta el 2014, era la Secretaría de Transporte y Vialidad (Setravi) ${ }^{4}$, creada en 1994, y que sustituyó, a su vez, a la Coordinación General de Transporte que había sido erigida diez años antes (1984), en reemplazo de la Comisión de Vialidad y Transporte Urbano (Covitur) que databa de 1978. Se puede decir que la creación de la Setravi obedece a la

4 Es importante precisar que en julio de 2014 el GDF promulgó la nueva Ley de movilidad del Distrito Federal, y además reemplazó a Setravi por la Secretaría de Movilidad. Sin embargo, para el trabajo seguiremos abordando el marco legal vigente en el 2005. 
necesidad de centralizar las funciones de manejo y control del transporte tanto de carga como de pasajeros en la entidad.

De allí que la competencia de la Setravi, al respecto del transporte público concesionado, se centre en tres niveles regulatorios que describimos a continuación:

El primero de los aspectos normativos está vinculado con "las especificaciones de los vehículos" (Islas 2000, 222) de transporte, el cual busca garantizar que el estado de los mismos brinde las mayores condiciones de seguridad y comodidad a los usuarios. Se establece, además, la vida útil de estos, de tal forma que no sobrepasen el límite establecido.

El segundo aspecto normativo se relaciona con "los límites de velocidad, condiciones de operación de los vehículos y requisitos de aptitud física de los conductores" (Islas 2000, 224). Se busca con ello garantizar que la operación de las unidades obedezca a parámetros claros, donde se conjunten las características mecánicas adecuadas con la pericia y preparación adecuada de los operadores.

El tercer aspecto normativo se refiere a cuestiones vinculadas con la integralidad del servicio: "aumento o disminución de la oferta, control del cálculo y aplicación de las tarifas, establecimiento de horarios y frecuencias, responsabilidad de las empresas ante posibles daños o pérdidas, regulación y orden de servicio" (Islas 2000, 224). En este apartado tienen asidero las dos consideraciones normativas anteriores, dado que el incumplimiento de estos factores previos tendrá una incidencia definitiva en el número de unidades disponibles para la operación, así como en las condiciones de control de los tiempos de despacho y, por lo tanto, en la frecuencia de circulación.

Ahora bien, para lograr comprender la configuración del transporte público en la ciudad de México, no basta con tener en cuenta los tres ámbitos ya citados; es necesario insertar la dinámica de prestación de este servicio en una discusión más amplia que se encuentra vinculada con lo que algunos estudiosos han denominado la ciudad informal. Este término pretende describir un proceso histórico y territorial en el cual se puede ubicar la matriz explicativa de la organización del espacio urbano.

La ciudad informal ha sido descrita como un fenómeno que no es exclusivo de la ciudad de México, sino de la realidad más amplia de los procesos de urbanización en países en desarrollo, particularmente en la segunda mitad del siglo pasado. De allí se desprende una informalidad que se manifiesta, por ejemplo, en la urbanización popular -aunque no exclusivamente en ella-, y más ampliamente en la configuración, como lo manifestó Clara Salazar, de un "campo de actividad" en el que encontramos una disposición de actores sociales que desarrollan una relación particular con el ámbito normativo y legal, y que impacta directamente en las prácticas y formas de apropiación del espacio (2012). Ahora, nuestro objeto es emplear el prisma de la ciudad informal para poder leer la forma en la que se configuró, desde esta misma lógica, una estructura institucional que tuvo como resultado la organización de las rutas de transporte público que le dieron paso, en las avenidas intervenidas, al BRT.

De acuerdo con Davis $(2012,15)$, la forma en la que los actores urbanos se vinculan con las "instituciones formales y los marcos reguladores" no describe una relación dicotómica en términos de lo formal versus lo informal. En su análisis, 
la autora llama la atención sobre la existencia de niveles de tolerancia a la informalidad por parte tanto de las autoridades como de los mismos ciudadanos implicados en determinada actividad -para nuestro caso el transporte público-. De allí que se pueda advertir la pervivencia de acuerdos informales a los cuales se acude para sortear situaciones cotidianas que, de pasar por los filtros establecidos por lo formal, no lograrían ser resueltas de manera expedita.

En dichos arreglos informarles tienen asidero las "negociaciones cotidianas" (Rincón 2006, 674), muchas de ellas al margen de la legalidad, sobre las que se erige la ciudad. Dichos acuerdos entre actores se orientan por la necesidad de llenar vacíos regulatorios o bien por la urgencia de adaptarse a un medio urbano que espacial, social y culturalmente se presenta con fuerte rasgos de exclusión y segregación.

Este marco de lo informal es "una base para la acción" (Davis 2012, 18) colectiva de los individuos urbanos que, en su afán por ganar visibilidad política en el concierto de la gobernabilidad capitalina, se ven abocados a desarrollar formas de acceso a los servicios, donde el factor mediador $-\mathrm{y}$ del acceso mismo- depende exclusivamente de mecanismos surgidos de su experiencia cotidiana, mas no de un ordenamiento legal, que aun existiendo no logra satisfacer la urgencia de movilizarse por la ciudad.

Esta línea de reflexión nos permite ubicar en contexto las concesiones de transporte público, y las prácticas de movilidad urbana que se relacionan con este modelo de organización que, si bien se encuentra normado, como lo resaltamos en un comienzo, obedece también a lógicas de negociación y generación de acuerdos informales que se traducen en instituciones de esta misma naturaleza.

El modelo de concesiones de transporte público urbano a operadores particulares se define en la LTVDF como el "Acto administrativo por virtud del cual, la Secretaría confiere a una persona física o moral la prestación del servicio público local de transporte de pasajeros o de carga, mediante la utilización de bienes del dominio público o privado del Distrito" (GDF 2002, 2). Los adjudicatarios de las concesiones se comprometen a la prestación del servicio de transporte con unas condiciones adecuadas de seguridad, cumplimiento y efectividad.

El complejo entramado de las concesiones de transporte público a particulares se puede evidenciar claramente en la existencia de diferentes niveles o grupos de actores que participan de las mismas. Así, de acuerdo con Nava y Ramírez, en primer lugar, se encuentran "los concesionarios, que son los dueños de los juegos de placas y los permisos para circular y operar"; en segundo lugar, se ubican "los dueños de las unidades, que no necesariamente son los dueños de las concesiones y las placas, y finalmente los choferes" de las unidades de transporte $(2008,415)$. De igual forma, dentro de cada ruta concesionada se puede percibir la existencia de "variantes o combinaciones" de intereses y propiedad $(2008,415)$.

De estas relaciones y combinaciones de actores dentro de cada ruta, surgen lazos de poder que han sido ampliamente documentados en trabajos como el Leviatán urbano de Diane Davis (1999) o Nadie está satisfecho de Ignacio López (1999). Ambos autores, desde perspectivas analíticas diferentes, concuerdan en señalar, como fundamento de la problemática del transporte, dos variables determinantes: la primera, marcada por el arreglo político entre las agremiaciones de transportistas -que se documenta desde el nacimiento de la Alianza de 
Camioneros de México en 1919 y las sucesivas organizaciones derivadas de ellay las autoridades del Distrito Federal; la segunda, vinculada con el crecimiento de la ciudad de México y la consecuente necesidad de atender la demanda de movilización de los habitantes a los espacios de actividad cotidiana, que no podía ser cubierta exclusivamente por los sistemas estatales de transporte -como es el caso del tranvía, en su período nacionalizado, el metro, la Ruta 100, y luego de ella la Red de Transporte de Pasajeros del Distrito Federal, en adelante RTP5 (Davis 1999).

Se conjugan, por tanto, las variables políticas con las territoriales para dar como resultado un esquema de transporte público concesionado en el cual el gobierno se halla cautivo por este y, por tanto, con estrechos márgenes para hacer cumplir los contenidos normativos formales. El punto más evidente de ello se puede encontrar, por ejemplo, en la designación que hace en 1971 el presidente Luis Echeverría Álvarez de Octavio Sentíes -representante legal de la Alianza de Camioneros- como regente de la ciudad de México (López 1999); con lo cual se demuestra tanto la supremacía y crecimiento sostenido del transporte dado en concesión como el poder político de las agremiaciones.

No obstante, cabe aclarar que el panorama político, institucional y, por tanto, los instrumentos de gestión urbana presentes en las décadas de la expansión del modelo concesionado difieren de las condiciones dadas al momento de la implementación del BRT. Así también, la ciudad de México ha sufrido transformaciones en su configuración territorial, aun cuando, como lo señalan Salazar e Ibarra (2006), en la actualidad se sigan manteniendo patrones históricos en el desplazamiento de los trabajadores de la ZMVM (Zona Metropolitana del Valle de México) que, como se infiere de las Encuestas Origen Destino de 1994 y 2007, son atendidos principalmente por las rutas concesionadas.

Retomando el proceso de configuración institucional del transporte concesionado, se puede advertir que el marco normativo formal no dejaría lugar a equívocos: los concesionarios cumplen con el servicio o de lo contrario estarían contraviniendo las leyes, situación que les acarrearía sanciones e incluso, como también lo contempla la norma, la suspensión de la concesión. Sin embargo -y en esto interviene uno de los factores de la informalidad-, dado que hay "una reducción paulatina de presupuesto gubernamental orientado al transporte" (Islas 2000, 211), desde la década de 1970 hasta la actualidad, los encargados de la prestación del servicio concesionado tienen amplios márgenes de maniobra que les permiten, además de erigirse como una fuerza política de primer orden, un manejo discrecional del marco legal.

No cabe duda, entonces, que el acomodo operativo de los concesionarios del transporte en relación con GDF está mediado por la incapacidad de este último para estructurar tanto un orden legal como medidas de cumplimiento que se reflejen, de forma directa, en las prácticas de movilidad. Lo anterior trae como consecuencia un esquema de funcionamiento del sector que en algunos casos se

5 "La Red de Transporte de Pasajeros del Distrito Federal (RTPDF) es un organismo de propiedad gubernamental (...) que opera, en el total del Distrito Federal, 100 rutas con una longitud de 3.061 kilómetros cuyo trazo parte de zonas periféricas y de bajos niveles de ingreso hacia las principales estaciones del Metro" (Nava y Ramírez 2008, 429). 
podría presentar como anárquico; en el que se conjugan la laxitud de las autoridades para aplicar las normas con las necesidades de movilidad de los usuarios, dando como resultado la comúnmente llamada "guerra por el pasaje" o "guerra por el centavo" (Hernández 2005).

Volvemos, con esto último, al esquema de negocio en el que la competencia por el pasaje es el recurso que tienen los transportistas para lograr unos niveles de utilidad que puedan cubrir los gastos asociados al servicio. Un aspecto que consideramos cardinal en este trabajo es el de los impactos o efectos que dicha organización tiene tanto en las prácticas cotidianas de los usuarios como en la propia organización del espacio, y justamente por ello hacemos hincapié en la informalidad que tiene su nicho de oportunidad en el transporte concesionado.

Dada la necesidad de darle cauce al análisis de las prácticas espaciales de los usuarios que se desplazaban por las avenidas que fueron intervenidas por el metrobús, en las siguientes páginas expondremos la forma en la que se completa el cuadro analítico del orden urbano asociado al modelo de rutas concesionadas. Para ello, se establecerán líneas de continuidad con los conceptos que describimos en la primera parte del artículo e, igualmente, integraremos en la discusión el problema de la informalidad, y cómo ella se manifiesta en las prácticas cotidianas de los usuarios.

\subsection{Las prácticas de movilidad}

Hasta el momento hemos centrado nuestra atención en las relaciones que establecen los concesionarios de transporte público con las autoridades del GDF mediadas por la normatividad formal existente; además, insertamos estas relaciones en la discusión de la ciudad informal como categoría de análisis que nos permite leer la forma en la que los actores asumen -o resignifican- y llevan a la práctica los postulados del marco normativo. Sin embargo, los usuarios no pueden ser abstraídos de lo anterior, pues son ellos quienes, en conjunción con los dos actores ya referidos, conforman, al decir de Clara Salazar (2012), un campo de actividad en el que el espacio urbano es tanto "el escenario de lo real", como un factor que "influye en la estructuración misma" de los procesos sociales (Lezama 2010, 32).

Con lo anterior, habría un punto de acuerdo de diversas disciplinas que han integrado el espacio como objeto de discusión tanto en el campo de lo epistemológico como en el del quehacer investigativo propio de cada una de ellas. Y aún con acentos particulares, se reconoce en la dimensión espacial un potente factor de análisis social, en su conjunto más amplio y en particular, en el entorno de lo urbano. De allí que al momento de hablar de interacciones, representaciones y prácticas culturales, el espacio, en sus diversas acepciones, esté inevitablemente presente.

Ahora bien, para lograr comprender la manera en la que se transforman las prácticas de movilidad de los pasajeros con la entrada en funcionamiento del metrobús, es necesario precisar que los sistemas de transporte, y en particular los BRT, están constituidos por un conjunto de operadores espaciales que son entendidos, de acuerdo con el Dictionnaire de la Géographie et de l'espace des 
sociétés, como una "realidad social, humana y/o no-humana, dotada de una capacidad de acción espacial" (Lévy et Lussault 2003, 150).

Siguiendo con la definición propuesta, conviene acotar que los operadores espaciales no solo están vinculados con objetos materiales, artefactos o dispositivos (estaciones, autobuses, máquinas de cobro o carriles confinados), sino también con la posibilidad que tienen los individuos organizados para actuar espacialmente. En tal sentido, un asunto como la variación en la forma de ordenar la frecuencia de circulación de las unidades de transporte, se convierte en operador espacial por cuanto este cambio actúa tanto sobre el espacio como en la experiencia y las prácticas de los usuarios.

Dicho de otro modo, la importancia de pensar el metrobús en términos de operadores espaciales estriba en el hecho de que su implementación no solo representa sacar de circulación unos autobuses "desbarajustados" y reemplazarlos por unidades articuladas o biarticuladas de "última tecnología”. Por el contrario, cada uno de los dispositivos -su materialidad y estructura funcional- representa una nueva realidad socio-espacial que transforma la manera en la que se construye el edificio de la movilidad urbana.

En tal medida, los operadores espaciales que configuran la columna vertebral del BRT son los siguientes ${ }^{6}$ :

1. La existencia de estaciones para el abordaje de las unidades: de acuerdo con la Guía de planificación del sistema BRT, las estaciones del sistema están compuestas por tres elementos principales: "1) Subparadas o plataformas; 2) Áreas de transición; y 3) Infraestructura de integración como senderos peatonales necesarios, espacios para vendedores, estacionamientos de bicicletas u otra actividad comercial" (ITDP 2010, 389).

2. El sistema de pago que se realiza por medio de un binomio de dispositivos ubicados a la entrada de las estaciones: máquinas de compra y recarga de tarjetas-torniquetes; junto a la implementación de este sistema automático de cobro, se puede apreciar la emergencia de nuevos actores: compañías encargadas de gestionar y administrar los cobros y recursos generados en la operación.

3. Las unidades de gran capacidad, generalmente articuladas y biarticuladas: los autobuses de la primera categoría tienen capacidad para transportar 160 pasajeros (40 sentados y 120 de pie), mientras para la segunda categoría la configuración es de 240 pasajeros ( 53 sentados y 187 de pie).

4. El carril confinado y exclusivo para la circulación de los autobuses del BRT: un carril confinado, dedicado o segregado (se emplean como sinónimos) es una intervención de naturaleza espacial, en la cual, por medio de diversos mecanismos, se separa la circulación de los autobuses del BRT de los demás vehículos o de otros modelos de transporte (Hidalgo 2010, 12).

5. El control de tiempos de despacho: ubicar este aspecto como un operador espacial tiene sentido en la medida en la que, independientemente de la

6 Estos operadores se definieron tomando en consideración documentos publicados por las dos principales organizaciones encargadas de la promoción de los sistemas BRT en América Latina: ITDP y EMBARQ. Además, se consultó bibliografía referente a la implementación de este modelo de transporte en Bogotá, Quito y Curitiba. 
configuración y del grado de articulación de los componentes estructurales de un modelo de transporte en particular, las frecuencias de circulación de sus unidades son, en último término, un ámbito en el que se manifiesta de forma clara la organización tanto del espacio urbano como de las prácticas que allí tienen cabida (CTS -Centro de Transporte Sustentable- 2009).

En el presente apartado, se describirán estos operadores en función de la estructura de las rutas de transporte concesionado que circulaban por la Avenida Insurgentes, en particular la Ruta 2. La información de este segmento proviene de entrevistas realizadas a los usuarios de la línea 1 del metrobús 7 .

En primer lugar, encontramos la falta de estaciones para el abordaje y descenso de las unidades de transporte público concesionado. Así, los usuarios de los microbuses o autobuses solicitaban su parada, y el conductor, de forma discrecional, decidía el lugar en el que lo hacía y los pasajeros hacían la señal de abordaje a lo largo de la vialidad, sin atender a la señalización de los lugares indicados para ello.

Esta práctica, que provenía de un acuerdo tácito entre pasajeros y operarios, estaba mediada, no por lo que dictaba el RTVDF, sino por condiciones vinculadas esencialmente con la competencia por el pasaje. De tal forma, aun cuando existiera voluntad de cumplimiento de la norma por parte de los usuarios o los conductores, emergían limitaciones funcionales que impedían el apego a las normas. Con esto nos referimos al hecho de que los controles sobre los tiempos de despacho de las unidades condicionan la ganancia de los operadores $y$, en consecuencia, los conductores deben asegurar un número determinado de pasajeros a lo largo del recorrido para así cumplir con las cuotas o costos de recuperación.

En este sentido, si consideramos los parabuses ${ }^{8}$ como dispositivos de naturaleza espacial que median en la prestación del servicio, es posible ver que su funcionalidad no está dada única y exclusivamente por su forma o materialidad, pues esta no tiene existencia más allá -o independiente- del uso que le dan los actores implicados en el transporte público. De tal suerte, en el caso del modelo de rutas concesionadas, el parabús, al insertarse en un esquema informal de funcionamiento, no tiene la posibilidad de regular por sí solo el ascenso y descenso de pasajeros.

Así, en tanto dispositivo, el parabús responde a los dos elementos que estructuran el orden urbano -la normatividad y las prácticas-, y la variación en alguno de estos ámbitos va a impactar de forma directa en el uso que se hace del mismo, como se verá en el caso del metrobús o como también se puede advertir en el funcionamiento de los sistemas de transporte que están a cargo del GDF como

7 El desarrollo de las entrevistas se hizo bajo la modalidad de recorridos-entrevista, en los cuales se acompañaba durante el viaje en metrobús a los pasajeros y allí se procedía a desarrollar los puntos establecidos en el guión. Es importante resaltar que los recorridos-entrevista contribuyeron a enriquecer el ejercicio, dado que fue posible que los mismos entrevistados validaran, a lo largo del trayecto, la información proporcionada. Así, fue constante que tuvieran expresiones como: "ahí lo ves", "mira cómo funciona esto", "no vayamos más lejos para entenderlo", "lo que te digo es cierto". Estas entrevistas se desarrollaron en la ciudad de México entre marzo y septiembre de 2012.

8 Lugares que están definidos y señalizados para fungir como paradas de los autobuses. 
el RTP o el trolebús, los cuales solo hacen sus paradas en los lugares señalados para tal caso.

Con el fin de establecer los elementos de comparación con las estaciones del sistema BRT, conviene decir que los paraderos de bus se encuentran configurados espacialmente como escenarios abiertos y flexibles, en los que la forma de interacción entre los actores remite a una lógica de lo informal, en la cual lo mismo pueden ser usados para esperar la llegada de las unidades, para guarecerse de las lluvias o para establecerse allí con un puesto de ventas de mercancías de diversa índole.

La anterior descripción de la práctica informal de abordaje y descenso de las unidades nos remite a una noción de eslabonamiento de factores explicativos del orden particular de funcionamiento del transporte concesionado, donde no es posible comprender lo aleatorio de este proceso sin atender al conjunto de las condiciones de competencia que rigen al servicio.

En segundo lugar, se encuentra el sistema de pago en los autobuses y microbuses de la Ruta 2, el cual tiene dos características esenciales: el abono del pasaje se realiza con el conductor o un ayudante contratado por este y la existencia de una tarifa diferencial según la distancia del recorrido. En tal medida, al momento de abordar las unidades de la Ruta 2 -las del RTP contenían una alcancía para depositar el valor del pasaje-, y disponerse a efectuar el pago por el viaje, los usuarios se encontraban no solo ante una transacción económica, sino ante una interacción personal con el conductor y eventualmente también con el ayudante del vehículo. En dicha relación entre pasajeros y operadores, se podía apreciar un complejo de situaciones asociadas al servicio, en las cuales la confianza y la experiencia no eran asumidas como cualidades absolutas sino contextuales o, en otros términos, dependientes de las condiciones cotidianas de transporte.

En cuanto a los usuarios frecuentes de la avenida Insurgentes, el conocimiento de su recorrido cotidiano les permitía conocer el valor del pasaje que debían abonar al conductor, por lo cual cuando este disponía un cobro más elevado, se apelaba generalmente a decir: "todos los días hago este trayecto y siempre me cobran lo que le estoy dando". Si continuaban las discrepancias en cuanto a la determinación de uno y otro, el conductor optaba por zanjar la discusión permitiendo que el pasajero continuara el viaje o le pedía que, de no estar de acuerdo con su decisión, descendiera del vehículo.

Sin embargo, este no era el único caso de interacción en el lugar del cobro. También se presentaba el caso de personas que, sabiendo el valor del trayecto recorrido, no llevaban el dinero necesario para cubrirlo. En este punto, el pasajero establecía un diálogo con el conductor -quien controla el servicio- para que le permitiera acceder por un valor menor al estipulado en las disposiciones de la Setravi. Generalmente, cuando la diferencia entre la suma a abonar y la disponible por parte del usuario no era de mayor cuantía -lo que indica que estaba tasaba en términos de centavos-, se cerraba el acuerdo y el usuario podía efectuar el viaje.

A las dos características del modelo de prestación del servicio de transporte a cargo de las rutas concesionadas, se suma un tercer elemento constituido por el tipo de unidades empleadas para esta función. Así, la ruta 2 que cubría el trayecto de San Ángel al CETRAM de Indios Verdes era atendida por unidades de 
autobuses y microbuses de mediana y baja capacidad que empezaron a circular por la ciudad en el segundo lustro de la década de 1980, lo cual indica que el tiempo de servicio de las mismas era de 15 años en promedio. Conviene mencionar que en esta senda también circulaban unidades del RTP, las cuales, con seis rutas hasta el 2004, atendían 38\% de la demanda de pasajeros (CTS 2005; 2009).

De acuerdo con López, la aparición de "minibuses con capacidad para 18 pasajeros sentados, con un servicio de lujo" $(1999,100)$ en sustitución de las combis, surge en el seno de las agremiaciones de transportistas como una polémica iniciativa que no hacía parte de un programa gubernamental orientado a regular el ya complejo panorama del transporte público en la ciudad. De tal suerte, los microbuses, en una carrera interminable por el control de las calles, invaden Ciudad de México y la hacen presa de su deficiente esquema de funcionamiento y operación.

De la anterior información se infiere que las 262 unidades correspondientes al modelo concesionado que atendían la demanda de pasajeros en la avenida Insurgentes en el 2005, no lo hacían apegadas al contenido normativo que dictaba, en líneas generales, los términos de seguridad, calidad y confort adecuados. Las razones de ello pueden encontrarse en la configuración informal de un modelo de rutas que descansa en el paulatino abandono por parte de las autoridades del GDF de las funciones de control y supervisión de la prestación de este y otros servicios urbanos.

El cuarto elemento de análisis está dado por el uso de la vialidad por parte de las rutas concesionadas. En este caso, podríamos decir, sin que el apelativo parezca exagerado, que se trataba de un verdadero escenario de "disputa por las calles" (López 1999, 55), donde los concesionarios acudían a la prestación del servicio de transporte público bajo un modelo de competencia regido por la "guerra del pasaje".

En consecuencia, puede percibirse que, dado un esquema de operación con un menor grado de controles y filtros, existía un margen de oportunidad más amplio para el desarrollo de acuerdos informales tanto entre operadores y usuarios como entre los primeros y las autoridades encargadas de vigilar la prestación del servicio. Por tanto, si bien se contaba con la presencia de los marcos reglamentarios formales, cobraban mayor validez los arreglos funcionales que los conductores de las unidades establecían en el día a día, y que en su permanencia configuraban acuerdos que posibilitaban la operación.

Lo anterior tenía lugar en la Avenida Insurgentes, donde antes de entrar en operación el metrobús, los carriles eran ocupados sin restricciones por las unidades de las rutas de transporte público; este panorama se hacía más complejo, toda vez que se debía maniobrar por entre taxis y automóviles particulares, que competían por un lugar en esta arteria de la circulación urbana. Si bien el número de vehículos de transporte concesionado era numeroso, su papel como factor ordenador del tráfico y de la vialidad no era totalmente visible, o por lo menos no iba más allá de las congestiones surgidas por sus particulares maniobras de descenso y ascenso de pasajeros.

9 Concepto desarrollado por Javier Hernández (2005), el cual hace referencia a la relación del conductor con su unidad de transporte; en ella, dadas las formas de propiedad y los ritmos de trabajo, se hace necesario laborar extensas jornadas para lograr unos niveles de rentabilidad que permitan la subsistencia. 
Aquí se podría hablar de una diferencia cardinal con el metrobús: la existencia de un carril confinado obliga a disponer de una serie de mecanismos, dispositivos y operadores que no permitan al tráfico mixto entorpecer la operación del BRT. Empero, bien podría establecerse una línea de continuidad entre lo que sucedía antes de 2005 y el actual panorama: para los concesionarios las calles son su territorio de acción y por supuesto de lucha; así, en el momento de la negociación con el GDF, su principal asunto o interés fue la defensa de algo que ellos consideraron, por derecho de antigüedad, suyo.

Finalmente, el quinto elemento comparativo está constituido por los tiempos de despacho de las unidades desde las bases de operación. Así, "los pasajeros se ven obligados a esperar el arribo de otros usuarios hasta que la capacidad del vehículo se complete y pueda iniciarse el viaje, lo que se traduce en una frecuencia de salida superior a los cinco minutos reglamentarios" (Islas 2000, 89). Esta situación descrita por Víctor Islas (2000) se encuentra inserta en la discusión de los métodos empleados por las rutas para definir la frecuencia en la que cada unidad debe realizar su recorrido: estos atienden principalmente al conocimiento, en el campo, de los horarios en los que hay mayor afluencia de usuarios por las vialidades que, si bien puede tazarse en términos de horas pico u horas valle, también obedece a particularidades de cada uno de los flujos atendidos.

De allí que a lo largo de los años de operación por determinadas vialidades se pueden encontrar, en términos institucionales, aprendizajes que dan sentido a las acciones de los actores. Por tanto, aun cuando se remarque el carácter informal de las prácticas de asociación entre los usuarios y los transportistas, es evidente que se generan márgenes de negociación que son aceptados y asumidos como válidos; lo cual se traduce en la relativa tolerancia a la espera de las unidades por parte de los pasajeros. Para ello, estos ubican cotidianamente sitios de parada que por su concurrencia son atendidos por los concesionarios con especial atención. Puede tratarse de zonas aledañas a las estaciones de metro o lugares cercanos a escenarios de gran atracción de viajes como centros corporativos, escuelas, sitios de espectáculos, plazas comerciales, etc.

Como una práctica derivada de estos acuerdos, se puede mencionar el desplazamiento de unidades vacías hacia estos lugares donde, por lo ya mencionado, se tiene asegurado el pasaje. Sin embargo, ello resta funcionalidad y regularidad a la prestación del servicio, toda vez que lo que prima, como parte del esquema de negocio concesionado, es la presurosa necesidad de garantizar un número mínimo de pasajeros sin atender a las condiciones de calidad del servicio, con el agravante de que la reglamentación prohíbe este tipo de acciones, pero justamente estas tienen cabida al ser prohijadas por un andamiaje institucional y de control que es débil en cuanto al cumplimiento de las normas.

Ahora bien, en lo que hemos dicho al respecto de este quinto operador, debe también reconocerse un elemento de continuidad con relación al planteamiento de las frecuencias en el funcionamiento del BRT: al igual que en el caso de las rutas concesionadas, fue necesario realizar un reconocimiento de los patrones de movilidad por las avenidas intervenidas para, con base en ello, realizar el trazado de las frecuencias de atención de la demanda, atendiendo, como ya se hacía, a la estructura diferenciada y, por lo mismo, no homogénea de los flujos de pasajeros a lo largo de los corredores. 
Por lo anterior, es fundamental decir que la distinción entre el modelo concesionado y el BRT debe encontrarse, de nueva cuenta, en la informalidad sobre la que descansa el primero y, por supuesto, en el subsiguiente impacto que ello tiene en la configuración de unas prácticas de movilidad. En este punto es pertinente hacer énfasis en que estos mecanismos de operación, aparentemente anárquicos o discrecionales, obedecen a una lógica de comportamiento de los actores, en la cual lo urbano se produce -y reproduce- desde la "incertidumbre (...) y no desde la certeza del derecho y la ley" (Giglia 2010, 355).

\section{Conclusiones}

Es claro que para comprender la estructura espacial del metrobús, se deben abordar las condiciones de las rutas de transporte público concesionado que circulaban por la Avenida Insurgentes antes de la puesta en marcha del BRT. Para darle cauce a esta tarea fundamental, es necesario partir de un recurso metodológico tanto en términos de la información necesaria como del recurso analítico. Por ello, los operadores espaciales se convierten en la pieza clave que se traza como hilo conductor de la argumentación a lo largo del texto.

$\mathrm{Al}$ respecto del orden urbano, es importante resaltar su carácter altamente dinámico y conflictivo, por cuanto se halla configurado a partir de prácticas de actores sociales que manifiestan sus intereses y asuntos desde la perspectiva de la acción concreta sobre la forma urbana, y allí el metrobús es un instrumento de lectura de este orden cambiante. Por tanto, se puede concluir que los objetos espaciales -de cualquiera naturaleza- dispuestos para el funcionamiento de las rutas de transporte concesionado y el BRT no tienen existencia per sé, lo cual nos lleva a pensar que se encuentran configurados por una dialéctica de forma (materialidad) y uso o socialización (práctica) que es mediada, a su vez, por las condiciones sociales, culturales y económicas de los individuos que habitan la ciudad.

En tal medida, el cambio en el espacio de socialización modifica tanto el tipo de interacciones atrás descritas como la naturaleza de los servicios a la movilidad (Monnet 2005) que brindaban las rutas de transporte concesionado, lo cual presupone la generación de nuevas dinámicas entre los usuarios, vale decir que mediadas ahora por la formalidad del sistema. Lo anterior nos lleva a inferir que el sentido de estar y ocupar un lugar se altera ostensiblemente, puesto que las estaciones del metrobús -a diferencia de lo que sucedía con los parabuses-, en cuanto a su forma y contenido normativo, acentúan la función del transporte público como mecanismo de enlace $y$, ante todo, un generador de flujos.

En síntesis, la existencia de normas -de carácter formal o convencional-, entrañan una lógica de direccionamiento que al leerse en el marco de acción del discurso del BRT en Ciudad de México permite conocer y desentrañar la manera en la que el GDF y las corporaciones promotoras del modelo buscan combatir el fuerte arraigo que tiene la informalidad urbana en el ámbito del transporte y la movilidad. Lo anterior cobra relevancia en el momento en que se ejecutan programas que, revestidos por el manto de la cultura ciudadana, desconocen la existencia de los llamados mundos sociales no previstos.

Por tanto, estos escenarios de acción estructurados por los actores involucrados manifiestan las limitaciones de un sistema que por la vía de la implementación de 
fuertes controles y filtros busca transformar de manera radical prácticas sociales que se legitiman no desde los códigos sino desde la necesidad de movilizarse por una ciudad que históricamente no ha contemplado la voz de los usuarios en la provisión de un servicio y derecho urbano primordial como el transporte.

Finalmente, si hablamos de prácticas sociales ancladas en el espacio, se debe reconocer que estas se construyen a partir de operadores y dispositivos espaciales que sirven como mediador entre las personas y los lugares. En este orden de ideas, el operador espacial no es una entidad que debe verse únicamente como parte del mobiliario urbano (Giglia 2010, 355); más bien, su contenido debe reconocerse en términos históricos como parte de acciones urbanísticas cambiantes.

\section{Referencias bibliográficas}

Borja, Jordi. «La ciudad es el espacio público». En Espacio público y reconstrucción de ciudadanía, de Patricia Ramírez, 59-88. México D.F.: UAM-Iztapalapa, 2003.

Bourdieu, Pierre. «Efectos de lugar». En La miseria del mundo, 119-124. Madrid: Akal, 1999.

CTS. «Viajar por el DF, un viacrucis». Movilidad amable, año $1, \mathrm{n}^{\circ} 1$ (septiembre de 2005): 44-47.

CTS (Centro de Transporte Sustentable). Metrobús: la fórmula ganadora. México D.F.: CTS, 2009.

Corrêa, Roberto. «Espaço: um conceito-chave da geografía». En Geografía: Conceitos e Temas, de Iná Casto et al., 15-47. Río de Janeiro: Bertrand, 1995.

Corrêa, Roberto. «Diferenciação sócio-espacial, escala e prácticas espaciais». Cidades, Vol. 4, no 6 (2007): 62-72.

Davis, Diane. El leviatán urbano. La ciudad de México en el siglo XX. México D.F.: Fondo de Cultura Económica, 1999.

Davis, Diane. «Fundamentos analíticos para el estudio de la informalidad: una breve introducción». En Informalidad urbana e incertidumbre. ¿Cómo estudiar la informalidad en las metrópolis?, de Felipe de Alba y Fréderic Lesamann, 12-37. México D.F.: PUEG-UNAM, 2012.

Duhau, Emilio y Angela Giglia. «Conflictos por el espacio y orden urbano». Estudios demográficos y ambientales, $\mathrm{n}^{\circ} 56$ (Mayo-Agosto 2004): 257-288.

Duhau, Emilio y Angela Giglia. Las reglas del desorden: habitar la metrópoli. México D.F.: UAM-Azcapotzalco, Siglo XXI Editores, 2008.

GDF (Gobierno del Distrito Federal). Ley de Transporte y Vialidad del Distrito Federal. México D.F.: GDF, 2002.

Giglia, Angela. «Producir y habitar la ciudad informal. Reflexiones desde la antropología». En Sistema mundial y nuevas geografías, de Miriam Alfie, 337-368. México D.F.: UAM-Azcapotzalco, 2010.

Giglia, Angela. El habitar y la cultura. Perspectivas teóricas y de investigación. Barcelona: UAM-Iztapalapa, Anthropos, 2012.

Giménez, Gilberto. Teoría y análisis cultural. México D.F.: Conaculta, 2005.

González, Salomón. La dimensión espacial del sistema automovil. Proyecto de investigación sobre movilidad en la ciudad de México. México D.F.: UAM-C, 2012. 
Hernández, Javier. «El transporte público concesionado». En El reto del transporte público en la ciudad de México. Voces, ideas y propuestas, de David Márquez Ayala, 30-52. México D.F.: Libros para todos, 2005.

Hidalgo, Darío. Lecciones aprendidas de mejoras en sistemas de autobuses de Latinoamérica y Asia. Modernización del transporte público. Washington D.C.: Embarq, 2010. Versión electrónica disponible en http://pdf.wri.org/modernizing_public_ transportation_es.pdf (último acceso: 30 de marzo de 2012).

Islas, Víctor. Llegando tarde al compromiso. México D.F.: El Colegio de México, 2000. ITDP (Institute for Transportation and Development Policy). Guía de planificación de los sistemas BRT. Autobuses de transito rápido. New York: ITDP, 2010. Versión electrónica disponible en http:/www.itdp.org/microsites/bus-rapid-transit-planning-guide/guia-de-planificacion-de-sistemas-brt-spanish/ (último acceso: 12 de febrero de 2012).

Lévy, Jacques et Michel Lussault. Dictionnaire de la geógraphie et de l'espace des sociétés. Paris: Belin, 2003.

Lezama, José. Teoría social, espacio y ciudad. México D.F.: El Colegio de México, 2010. López, Ignacio. Nadie está satisfecho. Los derroteros del transporte público concesionado en el D.F. México D.F.: Uteha, Acomex, 1999.

Monnet, Jerome. «Cruces comerciales: ambulantaje y servicios a la movilidad en la ciudad de México». Ponencia presentada en el Seminario Comercio y movilidades urbanas en tiempos de metropolización, México D.F., México, julio, 2005.

Nava, Emelina y Jaime Ramírez. «El funcionamiento del sector transporte y las posibilidades de negociación». En Construir ciudad. Un análisis multidimensional para los corredores de transporte en la Ciudad de México, de Clara Salazar y José Lezama, 407-463. México D.F.: El Colegio de México, 2008.

Rabotnikof, Nora. «Introducción: pensar lo público desde la ciudad». En Espacio público y construcción de ciudadanía, de Patricia Ramírez, 17-24. México D.F.: Flacso, 2003.

Rincón, Análida. «Racionalidades normativas y apropiación del territorio urbano: entre el territorio de la ley y la territorialidad de legalidades». Revista Economía, sociedad y territorio, $\mathrm{n}^{\circ} 20$, 2006: 673-702.

Salazar, Clara. «Los corredores confinados de transporte público en las metrópolis latinoamericanas: ¿una oportunidad para hacer ciudad?» En Construir ciudad. Un análisis multidimensional para los corredores de transporte en la ciudad de México, de Clara Salazar y José Lezama, 43-107. México D.F.: El Colegio de México, 2008.

Salazar, Clara y Valentín Ibarra. «Acceso desigual a la ciudad y movilidad». En Democracia y exclusión. Caminos encontrados en la ciudad de México, de Lucia Álvarez, 293-324. México D.F.: UNAM, 2006.

Santos, Milton. La naturaleza del espacio. Barcelona: Ariel, 2000.

Serfaty-Garzon, Perla. «L'Appropriation». En Dictionnaire de l'habitat et du logement, de Marion Segaud, 27-30. Paris: Editions Armand Colin, 2003.

Simmel, Georg. «Las metrópolis y la vida mental». Bifurcaciones, $\mathrm{n}^{\circ}$ 4, 2005: 1-10. Wirth, Louis. «El urbanismo como modo de vida». Bifurcaciones, $\mathrm{n}^{\circ}$ 2, 2005: 1-15.

\section{Entrevistas}

Clara Salazar, profesora-Investigadora del Colegio de México. Entrevistada por Madisson Carmona, septiembre 18 de 2012, Mexico D.F., México. 